\title{
Pressure Dependency Grüneisen Parameter $\gamma$ for bcc Mo
}

\author{
Chuanhui $\mathrm{Nie}^{1}$, Baochun Zong ${ }^{1} \&$ Junping Wang ${ }^{1}$ \\ ${ }^{1}$ School of Science, Beijing University of Civil Engineering and Architecture, Beijing, China \\ Correspondence: Chuanhui Nie, School of Science, Beijing University of Civil Engineering and Architecture, \\ Beijing 100044, China. Tel: 86-136-8302-8835. E-mail: sshns@sina.com
}

Received: May 15, 2014 Accepted: June 15, 2014 Online Published: June 16, 2014

doi:10.5539/apr.v6n4p26 URL: http://dx.doi.org/10.5539/apr.v6n4p26

\begin{abstract}
It has been reinforced by Kushwah et al. (Kushwah, 2013) that the Lindemann law doesn't work satisfactorily for the transition metals. We think this point is open to question. Combining the Lindemann law and the Vinet et al thermal equation of state with the melting curves of bcc Mo, we present an explicitly simple expression of the pressure dependency Grüneisen parameter $\gamma$ for Molybdenum. The results yield good agreement with density functional theory and experiments.
\end{abstract}

Keywords: Molybdenum, Grüneisen parameter, melting temperature

\section{Introduction}

The transition metal Molybdenum (Mo) has wide engineering and technology application for its thermal and mechanical strength and chemical resistance (Errandonea, 2001; Santamaría-Pérez, 2009; Belonoshko, 2008; Moruzzi, 1988). At low temperatures, Mo is stable in the body-centered-cubic (bcc) phase up to a pressure $(P)$ of about $700 \mathrm{GPa}$, where it transforms to fcc phase (Belonoshko, 2008). Considerable experimental and theoretical methods had been developed for Mo to study its thermodynamic properties such as melting temperature $T_{\mathrm{m}}$, thermal equation of state (EOS), Grüneisen parameter $\gamma$ and Debye temperature $\Theta_{\mathrm{D}}$, et al (Hixson, 1992; Zhang, 2008; Errandonea, 2005; Burakovsky, 2004; Kushwah, 2013; Zeng, 2011; Litasov, 2013; Al'tshuler, 1987; Asimow, 2009; Cazorla, 2007; Vinet, 1989). It's found that there exist great discrepancies in the melting curves between laser-heated diamond anvil cell (DAC) (Santamaría-Pérez, 2009) and shock-wave (SW) measurements (Hixson, 1992; Zhang, 2008). Errandonea (Errandonea, 2005) considered the following expression for the volume dependence of $\gamma$ similar to that proposed by Burakovsky and Preston (Burakovsky, 2004 ):

$$
\gamma=\gamma_{\infty}+\gamma_{1} \eta^{1 / 3}+\gamma_{2} \eta^{n}
$$

where $\eta=V / V_{0}$, the subscript " 0 " and " $\infty$ " represent the corresponding zero and infinite pressure, respectively, $\gamma_{1}, \gamma_{2}$ and $n$ are constants with $n>1$. However, the values of $\gamma_{1}, \gamma_{2}$ and $n$ proposed by Errandonea were different from those proposed by Burakovsky and Preston in Equation (1). Then the following volume dependence of melting curve (Equation (4) in Ref. (Errandonea, 2005) is different.

$$
T_{m}=T_{m 0} \eta^{-1 / 3} \exp \left[-6 \gamma_{1}\left(\eta^{1 / 3}-1\right)-\left(2 \gamma_{2} / n\right)\left(\eta^{n}-1\right)\right]
$$

Using the Lindemann law, Kushwah et al (Kushwah, 2013) had discussed curves of $\gamma$ vs $\eta$ and $T_{\mathrm{m}}$ vs pressure $P$ from Equations (1) and (2) for Mo. They found that the Burakovsky-Preston approach diverges the DAC experimental melting data while the Errandonea parameters reproduce it. But $\gamma$ becomes much smaller than 1 for the Errandonea approach which doesn't agree with the density functional theory performed by Zeng et al (Zeng, 2011).They then concluded that the Lindemann law doesn't work satisfactorily for the transition metals.

Litasov et al (Litasov, 2013) had discussed two other forms of $\gamma$ for Mo. One is from Mie-Gruneisen-Debye (MGD) EOS, $\gamma$ has the following form

$$
\gamma=\gamma_{0} \eta^{q}
$$

Fitting Equation (3) to the MGD relation yields $q=0.24$, which is different from the values 1.2 and 1.0 discussed by Zeng et al (Zeng, 2011). While $q=1.2$ and $q=1.0$, Equation (3) agrees with the results calculated by Zeng et al (Zeng, 2011) at low and high pressure, respectively. 
The other form of $\gamma$ is from Kunc-Einstein (KE) EOS (Litasov, 2013), and $\gamma$ has the following form by Al'tshuler et al (Al'tshuler, 1987):

$$
\gamma=\gamma_{\infty}+\left(\gamma_{0}-\gamma_{\infty}\right) \eta^{\beta}
$$

where $\gamma_{0}, \gamma_{\infty}$ and $\beta$ have different values for different models Mo-1 and Mo-2. It's found that (Litasov, 2013)the values of $\gamma$ derived from the model Mo-1 seem too high, whereas the model Mo-2 yielded consistent results with shock-wave (SW) data (Asimow, 2009) and theoretical calculations (Zeng, 2011).

In summary, while discussing the Grüneisen parameter for Mo, Equations (1) and (3) don't work satisfactorily. For Equation (4), parameters such as $\gamma_{0}, \gamma_{\infty}$ and $\beta$ in it are calculated by simultaneously weighted least-squares fitting to KE EOS (Litasov, 2013) from the available high-pressure P-V and P-V-T data, experimental data on the heat capacity, thermal expansion and adiabatic bulk modulus at zero pressure and various temperature. There are too many constants to be decided and the calculation is complicated. Therefore, it's necessary to propose a new form of $\gamma$ for bec Mo.

Here, we combined bcc melting curve with thermal equation of state and Lindemann law, and presented a new simple expression for the pressure dependence of the Grüneisen parameter $\gamma$ for bcc Mo. Method of analysis is shown in Section 2, results and discussions are presented in Section 3, and conclusions are made in section 4.

\section{Method of Analysis}

Recently, Carzorla et al (Cazorla, 2007) yielded the bcc Mo melting curve ( $P$ in GPa, $T_{\mathrm{m}}$ in $\mathrm{K}$ ) as follows

$$
T_{m}=T_{m 0}\left(1+\frac{P}{a}\right)^{b}
$$

where $T_{\mathrm{m} 0}=2894 \mathrm{~K}, a=37.2 \mathrm{GPa}$ and $b=0.43$. We noticed that Kushwah et al had compared the melting curve for Mo from the Lindemann law using the Burakovsky-Preston approach with the experimental DAC melting curve (Santamaría-Pérez, 2009). However, Belonoshko et al (Belonoshko, 2008) pointed out that the DAC melting curve of Mo at high $P$ is essentially a bcc-solid phase boundary, and that the original interpretation of the SW data on Mo is correct. We had plotted melting curves Equation (5) and experimental SW data (Hixson,1992; Zhang, 2008) in Fig. 1. It's found that Equation (5) fits good agreement with the experimental SW data.

Vinet et al (Vinet, 1989) demonstrated that the following two-parameter expression fits the experimental compression data for 24 different materials, including Mo, to within a few percent, over the entire experimentally attainable pressure range.

$$
\frac{\Delta V}{V_{0}}=\frac{\ln \left(1+C_{2} P\right)}{C_{1}}
$$

where $\Delta V=V_{0}-V, C_{1}=K_{0}^{\prime}+1, C_{2}=\frac{C_{1}}{K}, K_{0}, K_{0}^{\prime}$ denote the bulk modulus, pressure derivative of the bulk modulus at $P=0$, respectively. For Mo, $K_{0} \underline{\underline{K}}_{0} 268.5 \mathrm{GPa}, K_{0}^{\prime}=4$ (Kennedy, 1972).

The Lindemann melting law (Lindemann, 1910) is the widely used thermodynamic expression relating the melting temperature to the Grüneisen parameter. Different from Kushwah et al (Kushwah, 2013), we think that the Lindemann law does work for the transition metals.

$$
\frac{\mathrm{d} \ln T_{m}}{\mathrm{~d} \ln V}=\frac{2}{3}-2 \gamma
$$

Combining Equations (5),(6) with (7) we get

$$
\gamma=\frac{1}{3}+\frac{b K_{0}\left(1+C_{2} P\right)}{2(a+P)}\left[1-\frac{1}{C_{1}} \ln \left(1+C_{2} P\right)\right]
$$

\section{Results and Discussions}

(1) We have plotted $\gamma$ versus $P$ in Fig.2 according to the present work Equation (8). It's found that Equation (8) is in good agreement with the density functional theory (Zeng, 2011) and experiments (Hixson, 1992; Asimow, 2009). At $P=0$, our calculated zero-pressure value of $\gamma$ is 1.894 , which is consistent with the value $\gamma_{0}=1.8-2.0$ using MGD form (Litasov, 2013). At 390GPa, we obtained $\gamma=0.978$, agrees with the value 0.95 estimated by Hixson et al (Hixson, 1992) and 0.92 calculated by Zeng et al (Zeng, 2011). 
(2) Zeng et al (Zeng, 2011) had pointed out that the relative volume $\left(V / V_{0}\right)$ of bcc Mo is weakly dependent on $T$ from $0 K$ to $3500 K, \gamma$ shows very weakly dependency on temperature along the isobar, but its pressure dependence is strong, and it drops quickly as pressure increases. Equation (8) yields the same results.

(3) Both the well-known Birch-Murnaghan EOS (Birch, 1978) and the Vinet EOS (Vinet, 1987) show that at extreme compression $P \rightarrow \infty, V \rightarrow 0$. Equation (6) obviously dissatisfied this condition. We note that for bcc Mo, the maximum pressure is $700 \mathrm{GPa}$, far below the extreme compression. Therefore, Equation ( 6) is just used over a given pressure range. Saxena (Saxena, 2004) had also matched Equation (6) to Birch-Murnaghan EOS or Vinet EOS for iron, gold, platinum, bismuth and periclase, the results show a good consistency.

\section{Conclusions}

In the present work, we presented an explicitly new expression of the pressure dependency Grüneisen parameter $\gamma$ for bcc Mo while combining the melting curves with thermal equation of state and the Lindemann law. The method is simple and the results yield good agreement with density functional theory and experiments.

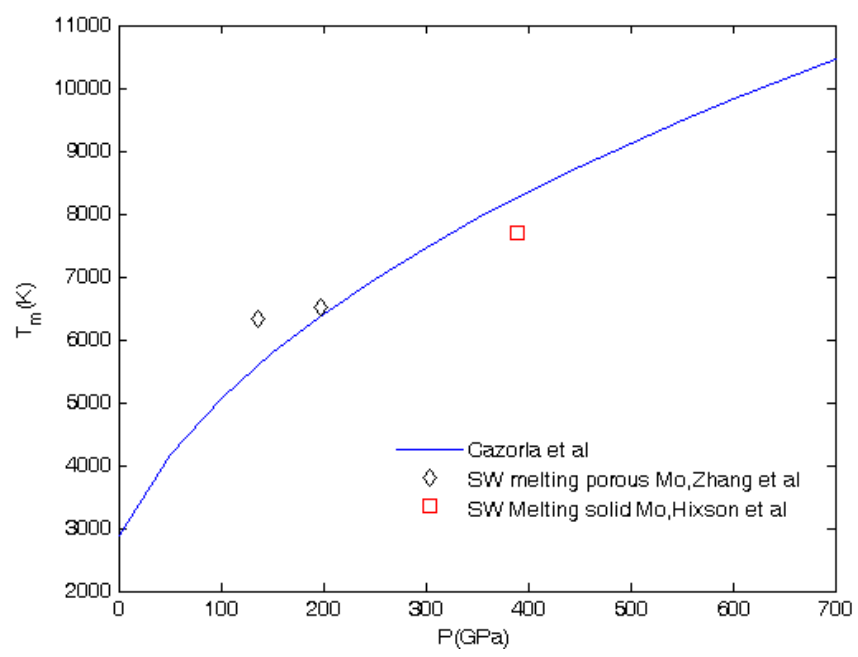

Figure 1. Melting temperature $T_{\mathrm{m}}$ versus $P$ for bcc Mo. The solid line corresponds to Equation (5) (Cazorla et al, 2007); ( $)$ SW data by Zhang et al. (2008); ( $\square$ ) SW data by Hixson and Fritz (1992)

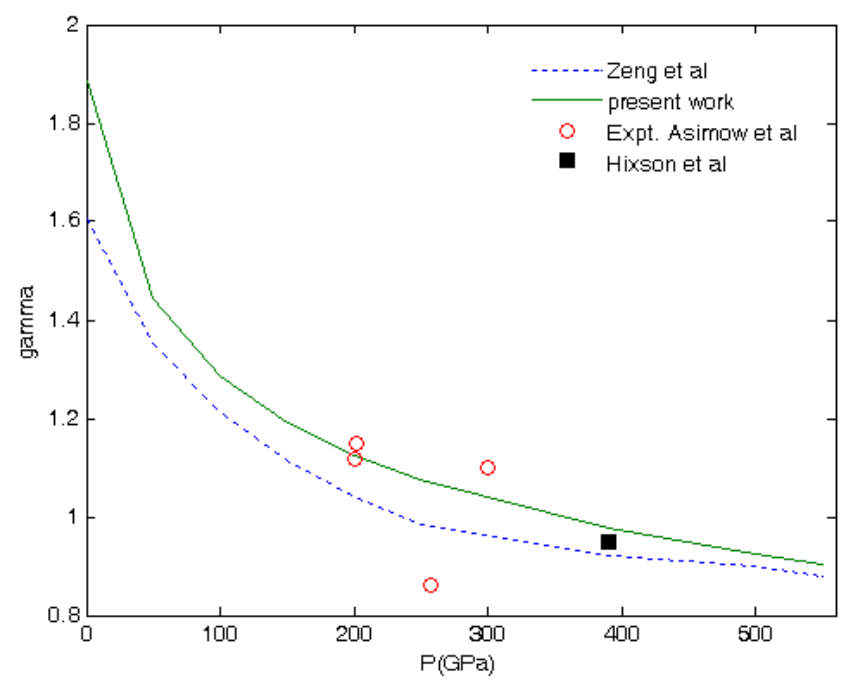

Figure 2. Variations of the Grüneisen parameter $\gamma$ with pressure $P$. The dotted line corresponds to theoretical calculations by Zeng, Z. Y. , Hu, C.E., Chen, X .R., et al. (2011). ( ○) Experimental data by Asimow, P. D., Sun D. Y. and Ahrens, T. J.. (2009). (匹) Hixson, R.S. and Fritz, J.N. (1992). Results discussed in the text Equation (8) are illustrated by the solid line 


\section{Acknowledgements}

Thanks for supports from Funding of Scientific Program of Beijing University of Civil Engineering and Architecture (2014), People's Republic of China. This work is also supported by Funding of Scientific Research of Beijing Municipality (051001607), People's Republic of China, and Funding of Scientific Research of Beijing University of Civil Engineering and Architecture (100903607), People's Republic of China.

\section{References}

Al'tshuler, L. V. S., Brusniskin, M., \& Kuz'menkov, E. A. (1987). Isotherms and Grüneisen functions of 25 metals. J. Appl. Mech. Tech. Phys., 28, 129-140. http://dx.doi.org/10.1007/BF00918785

Asimow, P. D., Sun D. Y., \& Ahrens, T. J. (2009). Shock compression of preheated molybdenum to 300GPa. Phys. Earth Planet Int., 174, 302-308. http://dx.doi.org/10.1016/j.pepi.2008.08.004

Belonoshko, A. B., Burakovsky, L., Chen, S. P., Johansson, B., Mikhaylushkin, A. S., Preston, D. L., ... \& Swift, D. C. (2008). Molybdenum at high pressure and temperature: melting from another solid phase. Physical review letters, 100(13), 135701. http://dx.doi.org/10.1103/PhysRevLett.100.135701

Birch, F. (1978). Finite strain isotherm and velocities for single-crystal and polycrystalline $\mathrm{NaCl}$ at high pressures and 300K. J.Geophys. Res., 83, 1257-1268. http://dx.doi.org/10.1029/JB083iB03p01257

Burakovsky, L., \& Preston, D. L. (2004). Analytic model of the Grüneisen parameter all densities. J. Phys. Chem. Solids, 65, 1581-1587. http://dx.doi.org/10.1016/j.jpcs.2003.10.076

Cazorla, C., Gillan, M. J., Taioli, S., \& Alfè, D. (2007), Ab initio melting curve of molybdenum by the phase coexistence method. J. Chem. Phys., 126(194502), 1-12.

Errandonea, D. (2005). Improving the understanding of the melting behaviour of Mo, Ta and $\mathrm{W}$ at extreme pressures. Physica B, 357, 356-364. http://dx.doi.org/10.1016/j.physb.2004.11.087

Errandonea, D., Schwager, B., \& Ditz, R. (2001). Systematics of transitional-metal melting. Phys. Rev. B, 63(132104), 1-4.

Hixson, R. S., \& Fritz, J. N. (1992). Shock compression of tungsten and molybdenum. J. Appl. Phys., 71, 1721-1728. http://dx.doi.org/10.1063/1.351203

Kennedy, G. C., \& Keeler, R. N. (1972). In D. E. Gray (Ed.), American Institute of Physics Handbook (3rd ed, pp. 4-38). New York: McGraw-Hill.

Kushwah, S. S., Tomar, Y. S., \& Upadhyay, A. K. (2013). On the volume-dependence of Grüneisen parameter and the Lindemann law of melting. J. Phys. Chem. Solids, 74, 1143-1145. http://dx.doi.org/10.1016/j. jpcs.2013.03.014

Lindemann, F. A. (1910). The calculation of molecular vibration frequencies. Phys. Z, 11, 609-612.

Litasov, K. D., Dorogokupets, P. I., \& Ohtani, E. (2013), Thermal equation of state and thermodynamic properties of molybdenum at high pressures. J. Appl. Phys., 113(093507), 1-10.

Moruzzi ,V. L., \& Janak, J. F. (1988). Calculated thermal properties of metals. Phys. Rev. B, 37, 790-799. http://dx.doi.org/10.1103/PhysRevB.37.790

Santamaría-Pérez, D., Ross, M., \& Errandonea, D. (2009). X-ray diffraction measurements of Mo melting to 119Gpa and the high pressure phase diagram. J. Chem. Phys., 130(124509), 1-8.

Saxena, S. K. (2004). Pressure-volume equation of state for solids. J. Phys. Chem. Solids, 65, 1561-1563. http://dx.doi.org/10.1016/j.jpcs.2004.02.003

Vinet, P., Rose, J. H., Ferrante J., \& Smith, J. R. (1989). Universal features of the equation of state of solids. J. Phys. Condens. Matter, 1, 1941-1963. http://dx.doi.org/10.1088/0953-8984/1/11/002

Vinet, P., Smith, J. R. , Ferrante, J., \& Rose, J. H. (1987). Temperature effect on the universal equation of state of solids. Phys. Rev. B, 35, 1945-1953. http://dx.doi.org/10.1103/PhysRevB.35.1945

Zeng, Z. Y., Hu, C. E., \& Chen, X. R. (2011). Density functional theory investigation of the phonon instability, thermal equation of state and melting curve of Mo, Phys. Chem. Chem. Phys., 13, 1669-1675. http://dx.doi.org/10.1039/c0cp01206h

Zhang, X. L., Cai, L. C., \& Chen, J. (2008). Melting Behaviour of Mo by Shock Wave Experiment. Chin. Phys. Lett., 25, 2969-2972. http://dx.doi.org/10.1088/0256-307X/25/8/064 


\section{Copyrights}

Copyright for this article is retained by the author(s), with first publication rights granted to the journal.

This is an open-access article distributed under the terms and conditions of the Creative Commons Attribution license (http://creativecommons.org/licenses/by/3.0/). 